\title{
Association analysis of $P O N 2$ genetic variants with serum paraoxonase activity and systemic lupus erythematosus
}

\author{
Sudeshna Dasgupta ${ }^{1}$, F Yesim Demirci ${ }^{1}$, Amy S Dressen ${ }^{1}$, Amy H Kao², Elisa Y Rhew ${ }^{3}$, Rosalind Ramsey-Goldman³
} Susan Manzi ${ }^{2,4}$, Candace M Kammerer ${ }^{1}$, M llyas Kamboh ${ }^{1 *}$

\begin{abstract}
Background: Low serum paraoxonase (PON) activity is associated with the risk of coronary artery disease, diabetes and systemic lupus erythematosus (SLE). Our prior studies have shown that the PON1/rs662 (p.Gln192Arg), PON1/ rs854560 (p.Leu55Met), PON3/rs17884563 and PON3/rs740264 SNPs (single nucleotide polymorphisms) significantly affect serum PON activity. Since PON1, PON2 and PON3 share high degree of structural and functional properties, in this study, we examined the role of PON2 genetic variation on serum PON activity, risk of SLE and SLE-related clinical manifestations in a Caucasian case-control sample.

Methods: PON2 SNPs were selected from HapMap and SeattleSNPs databases by including at least one tagSNP from each bin defined in these resources. A total of nineteen PON2 SNPs were successfully genotyped in 411 SLE cases and 511 healthy controls using pyrosequencing, restriction fragment length polymorphism (RFLP) or TaqMan allelic discrimination methods.

Results: Our pair-wise linkage disequilibrium (LD) analysis, using an $r^{2}$ cutoff of 0.7, identified 14 PON2 tagSNPs that captured all 19 PON2 variants in our sample, 12 of which were not in high LD with known PON1 and PON3 SNP modifiers of PON activity. Stepwise regression analysis of PON activity, including the known modifiers, identified five PON2 SNPs [rs6954345 (p.Ser311Cys), rs13306702, rs987539, rs11982486, and rs4729189; $P=0.005$ to $2.1 \times 10^{-6}$ ] that were significantly associated with PON activity. We found no association of PON2 SNPs with SLE risk but modest associations were observed with lupus nephritis (rs11981433, rs17876205, rs17876183) and immunologic disorder (rs11981433) in SLE patients ( $P=0.013$ to 0.042).

Conclusions: Our data indicate that PON2 genetic variants significantly affect variation in serum PON activity and have modest effects on risk of lupus nephritis and SLE-related immunologic disorder.
\end{abstract}

\section{Background}

Systemic lupus erythematosus (SLE), a systemic autoimmune disease with high female predominance (female to male ratio $=9: 1)$ in reproductive years (15-44 years), is characterized by a pronounced inflammation and the production of a variety of autoantibodies against multiple antigens. SLE pathogenesis is influenced by several genes, hormones and environmental agents. Familial aggregation and high heritability (up to 66\%) suggest

\footnotetext{
* Correspondence: kamboh@pitt.edu

'Department of Human Genetics, University of Pittsburgh, Pittsburgh, PA, USA

Full list of author information is available at the end of the article
}

that the genetic elements have strong influence on SLE predisposition $[1,2]$.

A prime cause of high mortality rate in SLE women is attributed to an unusually high rate of premature coronary artery disease (CAD) with risk of myocardial infarction 50 times higher than the general population [3]. The paraoxonase $(P O N)$ genes have received major attention as antioxidants that attenuate oxidation of low density lipoprotein (LDL), a key regulator in the pathogenesis of atherosclerosis leading to several cardiovascular diseases [4]. Low serum paraoxonase (PON) activity (using paraoxon as a substrate) is a significant risk factor for CAD $[5,6]$. Low PON activity is also associated
Ciomed Central

() 2011 Dasgupta et al; licensee BioMed Central Ltd. This is an Open Access article distributed under the terms of the Creative Commons Attribution License (http://creativecommons.org/licenses/by/2.0), which permits unrestricted use, distribution, and reproduction in any medium, provided the original work is properly cited. 
with SLE risk, independent of other known risk factors $[7,8]$. PON activity is under genetic control and the variants in the PON1 gene have strong influences on PON activity variation $[7,9]$.

PON2 is a positional candidate for SLE risk as the entire PON locus comprising three genes (PON1, PON2 and $P O N 3$ ) on chromosome 7q21.3 lies close to a linkage peak for SLE (LOD score of 2.40) in the same region $(7 \mathrm{q} 21-22)[10,11]$. All $P O N$ genes are antioxidants and can hydrolyze a variety of substrates $[4,12]$. PON1 and PON3 are mainly expressed in the liver and their protein products circulate in the blood as HDL-bound, while PON2 is ubiquitously expressed in human tissues and its product remains mostly intracellular as membrane-bound [4]. PON2 gene spans nearly $30 \mathrm{~kb}$ on chromosome 7 with 9 exons encoding two protein isoforms of 354 (NCBI Reference Sequence: NP_000296.2) and 342 (NCBI Reference Sequence: NP_001018171.1) amino acids [10]. Two common nonsynonymous PON2 coding variants have been reported to date [rs11545941 (p.Ala148Gly) and rs6954345 (p.Ser311Cys)] and the latter has been hypothesized to be a catalytic center for the hydrolysis of oxidized lipids [13]. So far, the PON2 reports mainly examined these two coding polymorphisms, of which the rs6954345 (p.Ser311Cys) SNP was found to be associated with several cardiovascular complications [14-16] and renal dysfunction [17], whereas the rs11545941 (p.Ala148Gly) SNP associated with plasma lipoprotein levels in some studies [18]. Recently, the PON2 protein was reported to degrade and inactivate $N$-3-oxododecanoyl-homoserine lactone, the quorum sensing signal of pathogenic bacteria [19] but its exact physiologic or pathophysiologic role has yet to be identified. PON2 protein shows highest lactonase activity among all PON proteins and the rs6954345 (p. Ser311Cys) SNP in recombinant PON2 was recently shown to cause altered glycosylation and impaired lactonase activity [20].

Our previous reports with SLE datasets have identified low PON activity as an independent risk factor for SLE and our analysis of PON1 and PON3 SNPs revealed some modest associations with lupus nephritis $[7,21]$. The PON1 and PON3 SNPs account for $30-40 \%$ of serum PON activity variation in Caucasians $[7,21]$. Given that PON1-2-3 gene cluster resides close to a linkage peak for SLE and our previous observations suggesting a relationship between SLE and PON activity/ PON1-3 genetic variation, the purpose of this study was to investigate the remaining member of the $P O N$ multigene family, PON2. We genotyped and analyzed 19 highly informative PON2 SNPs selected from HapMap and SeattleSNPs databases in a Caucasian SLE casecontrol sample. To our knowledge, this is the first comprehensive association study of the PON2 variants with risk of SLE, SLE-related clinical features and serum PON activity.

\section{Methods \\ Subjects}

A total of 411 Caucasian women with SLE and 511 Caucasian healthy women with no apparent history of SLE were studied. Peripheral blood samples were collected at the baseline visit and used for genotyping and measurement of serum PON activity (at the Pittsburgh site only) as described previously [7].

Of 411 SLE cases, 344 belonged to the Pittsburgh Lupus Registry and the rest $(n=67)$ to the Chicago SOLVABLE study (Study of Lupus Vascular and Bone Longterm Endpoints). All controls enrolled in our study were geographically matched to the cases: 454 controls were recruited from the Central Blood Bank of Pittsburgh and the rest $(n=57)$ from the Chicago SOLVABLE study. Demographic and clinical characteristics of SLE patients and controls are presented in Table 1 and described in detail elsewhere [22,23]. All SLE subjects were adult women aged 18 years and older and met the 1982 [24] and 1997 [25] revised American College of Rheumatology (ACR) classification criteria for SLE. The subset of the ACR criteria that were included in association analysis are shown in Table 1 [highly frequent (i.e. Arthritis and ANA) or infrequent (i.e. Neurologic disorder) subphenotypes were not suitable for a meaningful analysis given the size of our sample]. Also included in Table 1 is the percentage of SLE cases who had experienced one or more of physician-confirmed cardiac and vascular events (myocardial infarction, coronary artery bypass graft surgery, percutaneous transluminal coronary angioplasty, angina pectoris, cardiac death, stroke, transient ischemic attack, congestive heart failure, blood clots, or vascular surgery) in a subset of the SLE sample in which this data was available $(\mathrm{n}=292)$. The mean \pm standard deviation (SD) age was $43.5 \pm 11.4$ years for cases and $45.7 \pm 12.9$ years for controls. This study was approved by the institutional review board and all subjects gave written, informed consent.

\section{Selection of PON2 SNPs and genotyping}

We initially selected a total of twenty highly informative PON2 SNPs [residing within PON2 or 1-kb flanking regions and with $\geq 4 \%$ minor allele frequency (MAF)], by using the LD and tagSNP information available for Caucasians in the International HapMap http://www. hapmap.org and SeattleSNPs http://pga.gs.washington. edu/ databases (Figure 1). The tagSNP bins identified in these 2 databases were not identical (likely due to the sampling and methodological differences), thus we selected at least one tagSNP from each bin defined in these resources. One SNP (PON2/rs17876195) could not 
Table 1 Characteristics of the study population

\begin{tabular}{|c|c|c|c|}
\hline & Cases $(n=411)$ & Controls $(n=511)$ & Combined $(n=922)$ \\
\hline Mean age & $43.54 \pm 11.36$ & $45.68 \pm 12.86$ & $44.68 \pm 12.22$ \\
\hline Body mass index $\left(\mathrm{kg} / \mathrm{m}^{2}\right)$ & $26.90 \pm 6.82$ & $27.34 \pm 6.59$ & $27.14 \pm 6.70$ \\
\hline Current and/or Former Smoker (\%) & $37.84 \%$ & $40.29 \%$ & $39.23 \%$ \\
\hline PON activity (in units/liter)* & $613.88 \pm 412.94$ & $709.85 \pm 496.39$ & $671.44 \pm 466.87$ \\
\hline \multicolumn{4}{|l|}{ SLE manifestations $(\%)^{* *}$} \\
\hline Skin rash (malar and/or discoid) & $55 \%$ & - & - \\
\hline Photosensitivity & $63 \%$ & - & - \\
\hline Oral ulcers & $54 \%$ & - & - \\
\hline Serositis (pleuritis or pericarditis) & $44 \%$ & - & - \\
\hline Renal involvement & $30 \%$ & - & - \\
\hline Hematologic involvement*** & $54 \%$ & - & - \\
\hline Immunologic involvement**** & $72 \%$ & - & - \\
\hline Cardiac and vascular events ${ }^{* * * * *}$ & $33 \%$ & - & - \\
\hline
\end{tabular}

${ }^{*}$ Available only in a subset of the sample (291 cases and 436 controls).

**The subset of American College of Rheumatology (ACR) classification criteria for SLE that were included in association analysis.

***Hemolytic anemia or leukopenia (on two or more occasions) or lymphopenia (on two or more occasions) or thrombocytopenia.

****Anti-DNA antibody or anti-Sm antibody or positive finding of antiphospholipid antibodies.

*****Data available only in a subset of SLE patients (292 cases).

be genotyped due to technical difficulties, therefore a total of 19 successfully genotyped PON2 SNPs were included in our analyses.

Genomic DNA was isolated from the buffy coat using the QIAamp DNA kits (QIAGEN, Chatsworth, CA). Ten PON2 SNPs (rs11982486, rs2286233, rs17876116, rs11545941 (p.Ala148Gly), rs17876193, rs3735586, rs10261470, rs9641164, rs13306702 and rs17876183) were genotyped by Pyrosequencing using the PSQ HS 96 system (Biotage, Uppsala, Sweden) and the corresponding PCR and sequencing primers were provided in the Additional File 1, Table S1.

One of the PON2 coding SNPs, PON2/rs6954345 (p. Ser311Cys), was genotyped by restriction fragment length polymorphism (RFLP) analysis using the restriction enzyme DdeI. The PCR primers (forward = 5'AACAGGGCTTATTGATGATTGAGT-3' and reverse = 5' -ACAGACCCATTGTTGGCATAA-3') amplified a 145 bp product containing the SNP of interest. DdeI digestion of this product yielded a single fragment of 145 bp for GG genotype (CysCys), 2 fragments of 100 and 45 bp for CC genotype (SerSer), and 3 fragments of 145, 100 and $45 \mathrm{bp}$ for the heterozygous CG genotype (SerCys).

The remaining eight PON2 SNPs (rs2299267, rs12534274, rs987539, rs4729189, rs11981433, rs12704795, rs1639 and rs17876205) were genotyped by TaqMan allelic discrimination on an ABI 7900HT instrument (Applied Biosystems, Foster City, CA) using the following SNP genotyping assays: C___2630173_1_ for PON2/rs2299267, C_31373224_10 for PON2/rs12534274, C__8952813_10

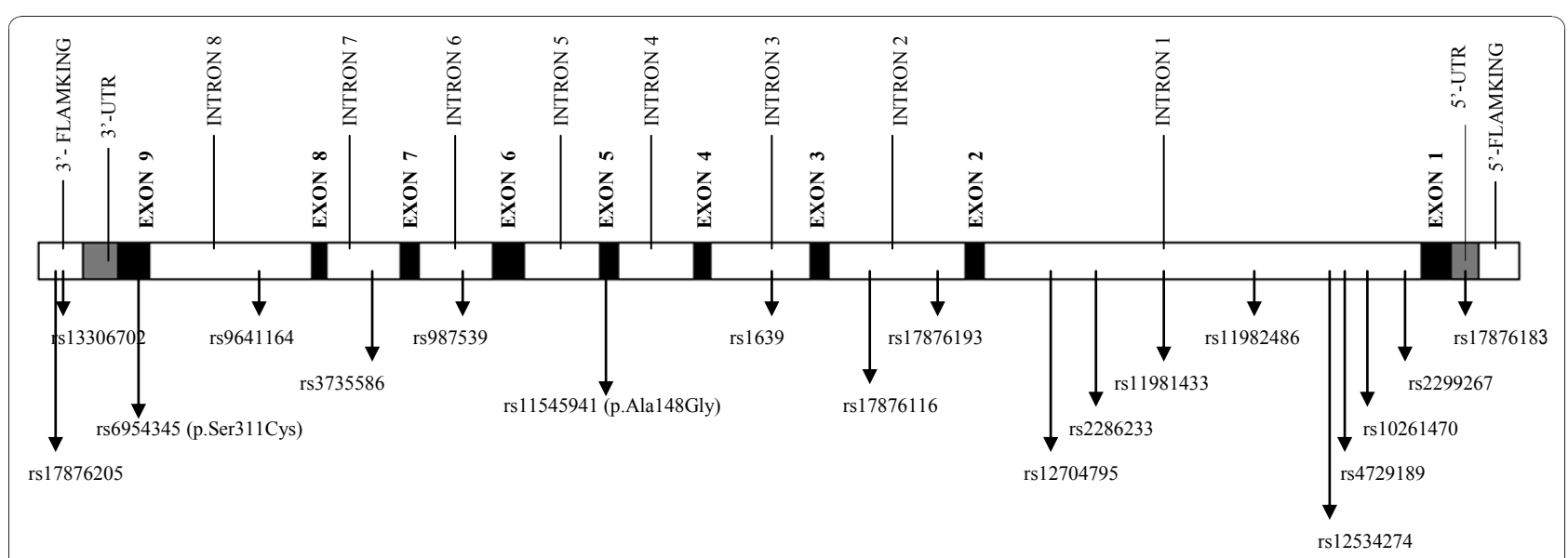

Figure 1 Locations of the genotyped 19 PON2 SNPs. The exons are depicted as black bars (UTRs are highlighted as gray) and introns \& flanking regions as white bars. 
for PON2/rs987539, C__27922117_10 for PON2/ rs4729189, C__2630169_10 for PON2/rs11981433, C_26570646_10 for PON2/rs12704795, C__11708890_10 for PON2/rs1639, and C__59001801_10 for PON2/ rs17876205.

\section{Statistical analysis}

Allele frequencies were calculated by allele counting and all SNPs were tested for deviations from HardyWeinberg Equilibrium (HWE) expectations. Haploview http://www.broadinstitute.org/mpg/haploview was used to assess the pairwise LD between all PON2 SNPs as well as the two PON1 SNPs [PON1/rs662 (p.Gln192Arg) and $P O N 1 /$ rs 854560 (p.Leu55Met)] and the two PON3 SNPs (PON3/rs740264 and PON3/rs17884563) that we previously reported to influence PON activity.

Data on PON activity was available only at the Pittsburgh site and the raw measurements were square root transformed to reduce non-normality. We conducted combined (forward and reverse) stepwise and multiple regression analyses to test for independent association of PON activity with environmental covariates [disease status, age, body mass index (BMI), smoking] [7] as well as the two PON1 SNPs [PON1/rs662 (p.Gln192Arg) and PON1/rs854560 (p.Leu55Met)], one PON3 SNP (PON3/rs17884563) and 13 PON2 SNPs that were in low LD. Allele effects were modeled as additive for common SNPs and as dominant for relatively uncommon variants. To determine whether additional, unmeasured genetic variation in PON2 might also influence PON activity, we also evaluated the association of PON2 haplotypes with PON activity.

Association of SLE status with PON2 SNPs was evaluated using logistic regression and the recruitment site (Pittsburgh or Chicago) and age (found to be significant in stepwise regression analysis of potential covariates examined, including the recruitment site, age, BMI and smoking) were included as covariates in the model. SNP effects were modeled as additive for common variants and dominant for relatively uncommon ones. Within SLE cases, the logistic regression analysis was performed to test for association between PON2 SNPs and SLErelated subphenotypes [skin involvement (malar rash and/or discoid rash), lupus nephritis, photosensitivity, oral ulcers, serositis, immunologic disorder and hematologic involvement] using a dominant model and including recruitment site and age as covariates.

All regression and haplotype analyses were performed using the R Statistical Suite (R Foundation for Statistical Computing, Vienna, Austria). Power analysis was performed using the Quanto program http://hydra. usc.edu/GxE.

\section{Results}

Pair-wise LD analysis of PON2 SNPs and relevant PON1 and PON3 SNPs

For all 19 PON2 SNPs, the observed minor allele frequencies (MAFs) in our control sample were similar to those reported in the HapMap database for Caucasians. The observed genotype frequencies of all PON2 SNPs were in accordance with HWE. Pairwise LD analyses revealed similar patterns in cases and controls, hence LD was computed by combining these two groups (Figure 2). Significant correlations $\left(r^{2} \geq 0.7\right)$ were found between five PON2 SNP pairs [PON2/rs6954345 (p.Ser311Cys) \& rs11545941 (p.Ala148Gly), $r^{2}=0.81$ )], $\left[P O N 2 / \mathrm{rs} 6954345\right.$ (p.Ser311Cys) \& rs12534274, $r^{2}=$ 0.72], [PON2/rs6954345 (p.Ser311Cys) \& rs3735586, $r^{2}$ $=0.92], \quad\left[\mathrm{rs} 1639 \& \mathrm{rs} 9641164 ; r^{2}=0.87\right]$ and [rs11981433 \& rs12704795; $\left.r^{2}=0.98\right]$. Hence, one of the SNPs from each of these 5 highly correlated SNP pairs [rs11545941 (p.Ala148Gly), rs12534274, rs3735586, rs1639, and rs12704795] were excluded from subsequent analyses. Although all 19 PON2 SNPs were reported as tagSNPs in at least one of the two publicly available databases using different $r^{2}$ cutoffs (HapMap and SeattleSNPs), a total of 14 tagSNP bins were identified in our sample using an $r^{2}$ cutoff of 0.7 .

Because PON1, PON2 and PON3 genes lie adjacent to each other and PON1 and PON3 variants are known modifiers of PON activity, we also assessed LD between the PON2 variants and the four PON1 and PON3 SNPs that were previously found to be associated with PON activity (Figure 2). Significant correlations $\left(r^{2} \geq 0.7\right)$ were observed between PON2/rs6954345 (p.Ser311Cys) and PON3/rs740264 $\left(r^{2}=0.77\right)$ and between PON2/ rs 17876193 and $P O N 3 /$ rs $17884563\left(r^{2}=0.81\right)$. From the first correlated SNP pair, PON2/rs6954345 (p. Ser311Cys) was included in subsequent analysis for PON activity (regression and haplotype) since this SNP had also tagged three other PON2 SNPs [rs11545941 (p. Ala148Gly), rs12534274 \& rs3735586]. From the second correlated SNP pair, PON3/rs17884563 was included in subsequent analysis of PON activity.

\section{PON2 tagSNPs and serum PON activity}

Table 2 shows the $P$ and $\mathrm{R}^{2}$ values from stepwise linear regression analysis of PON activity with PON2 tagSNPs along with the significant PON activity modifiers from PON1 [7] and PON3 [21] genes and possible environmental covariates (disease status, age, BMI, and smoking status). In addition to previously reported effects of disease status, age, and the PON1 and PON3 SNPs, we identified associations with 5 PON2 SNPs: rs13306702 $(P=$ 0.005), PON2/rs6954345 (p.Ser311Cys) $\left(P=2.1 \times 10^{-6}\right)$, 


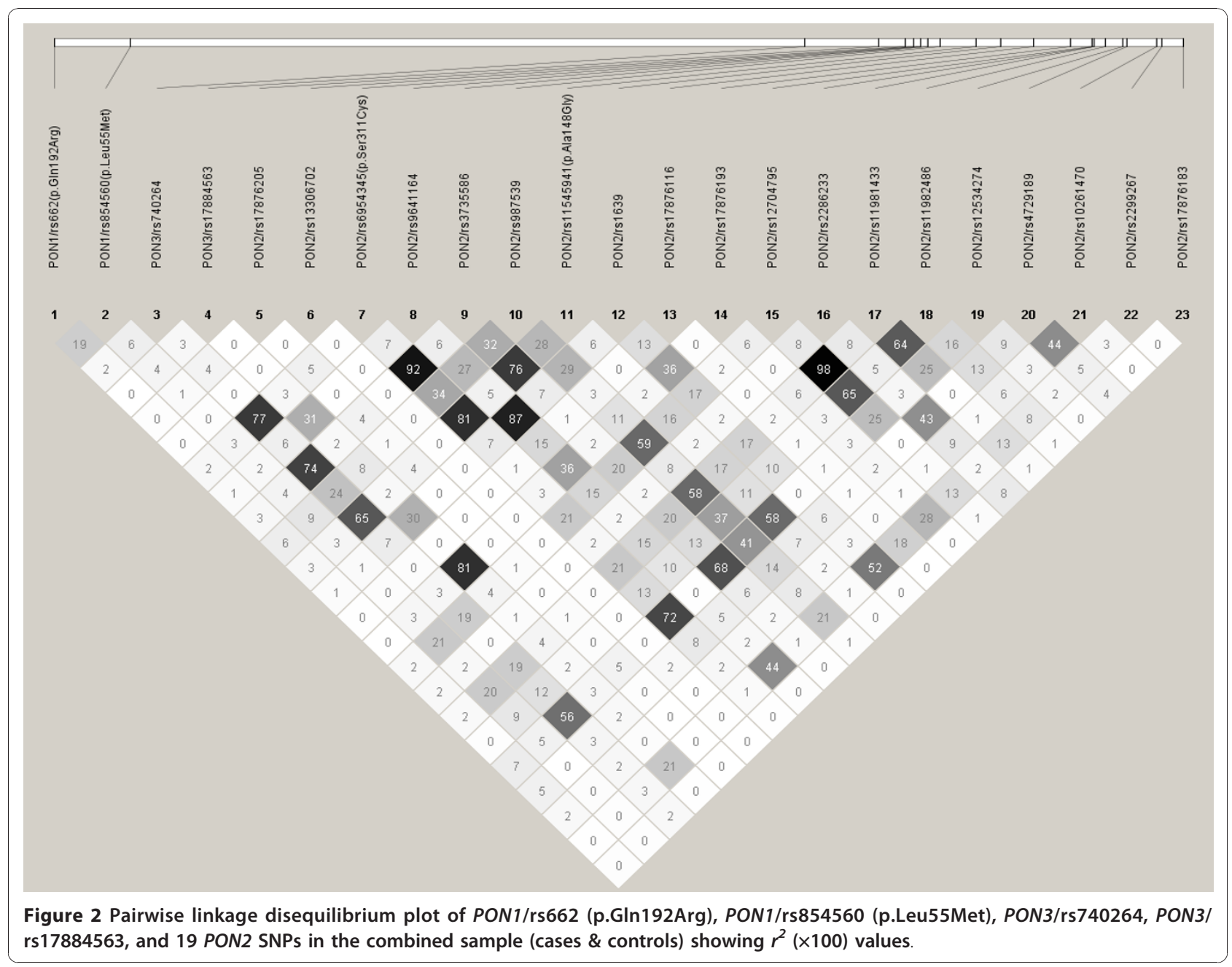

rs987539 $\left(P=3.4 \times 10^{-5}\right), \operatorname{rs} 11982486\left(P=7.0 \times 10^{-4}\right)$, and rs4729189 $(P=0.003)$. The same environmental covariates and SNPs were significant when all covariates and SNPs were entered in the model simultaneously. The

Table 2 Significant results obtained from stepwise linear regression analysis of serum PON activity (square root transformed) with SLE disease status, age, BMI, smoking, PON1/rs662 (p.Gln192Arg), PON1/rs854560 (p.Leu55Met) and $P O N 3 /$ rs 17884563 SNPs and 13 PON2 tagSNPs

\begin{tabular}{lcccc}
\hline FACTOR & MAF & Beta & $\boldsymbol{P}$ & $\boldsymbol{R}^{\mathbf{2}}$ \\
\hline disease & & -2.22 & $3.8 \times 10^{-8}$ & 0.015 \\
age & & 0.04 & 0.013 & 0.003 \\
PON1/rs662 (p.Gln192Arg) & 0.280 & 10.54 & $<2.0 \times 10^{-16}$ & 0.392 \\
PON1/rs854560 (p.Leu55Met) & 0.358 & -1.96 & $1.1 \times 10^{-8}$ & 0.017 \\
PON3/rs17884563 & 0.092 & -1.03 & 0.082 & 0.001 \\
PON2/rs13306702 & 0.017 & 3.87 & 0.005 & 0.004 \\
PON2/rs6954345 (p.Ser311Cys) & 0.233 & 2.22 & $2.1 \times 10^{-6}$ & 0.011 \\
PON2/rs987539 & 0.454 & -3.64 & $3.4 \times 10^{-5}$ & 0.009 \\
PON2/rs11982486 & 0.330 & -2.84 & $7.0 \times 10^{-4}$ & 0.006 \\
PON2/rs4729189 & 0.223 & -2.61 & 0.003 & 0.004 \\
\hline
\end{tabular}

beta values listed in Table 2 represent the effect of the minor allele of each of 5 PON2 SNPs on PON activity (square root transformed). PON activity increased with the presence of minor alleles of PON2/rs6954345 (p. Ser311Cys) and PON2/rs13306702 SNPs, and decreased with the presence of the minor alleles of the remaining 3 significant PON2 SNPs. Altogether, these 5 PON2 SNPs explained $3.4 \%$ of the variation in PON activity.

To assess whether unmeasured variants might influence PON activity, we also performed haplotype analysis for PON activity (Table 3). Because of the strong effects of PON1 and PON3 SNPs on PON activity, we performed haplotype analysis after adjusting for disease status, age, BMI, smoking, PON1/rs662 (p.Gln192Arg), PON1/ rs 854560 (p.Leu55Met), and PON3/rs17884563. Only one haplotype, Hap 6, was significantly associated with increased PON activity $\left(P=6.1 \times 10^{-5}\right)$ compared to the most common haplotype (Hap Base). This haplotype contained the minor allele for PON2/rs6954345 (p. Ser311Cys) that is the most significant of the PON2 variants. Therefore, this result is consistent with our 
Table 3 Haplotype analysis of 13 PON2 tagSNPs in relation to PON activity adjusted for disease status, age, BMI, smoking, PON1/rs662 (p.GIn192Arg), PON1/rs854560 (p.Leu55Met), and PON3/rs17884563

\begin{tabular}{|c|c|c|c|c|c|c|c|c|}
\hline & \multicolumn{8}{|c|}{ Haplotypes } \\
\hline & Hap 1 & Hap 2 & Hap 3 & Hap 4 & Hap 5 & Hap 6 & Hap Rare & Hap Base \\
\hline PON2/rs17876205 & G & G & G & G & G & G & * & G \\
\hline PON2/rs13306702 & G & G & G & G & G & G & * & G \\
\hline PON2/rs6954345 (p.Ser311Cys) & C & C & C & C & C & G & * & C \\
\hline PON2/rs9641164 & A & A & A & $\mathrm{T}$ & $\mathrm{T}$ & A & * & A \\
\hline PON2/rs987539 & C & C & C & $\mathrm{T}$ & $\mathrm{T}$ & $\mathrm{T}$ & * & C \\
\hline PON2/rs17876116 & G & G & G & G & G & G & * & G \\
\hline PON2/rs2286233 & A & $\mathrm{T}$ & $\mathrm{T}$ & A & A & A & * & A \\
\hline PON2/rs11981433 & C & $\mathrm{T}$ & $\mathrm{T}$ & $\mathrm{T}$ & $\mathrm{T}$ & $\mathrm{T}$ & * & C \\
\hline PON2/rs11982486 & $\mathrm{T}$ & $\mathrm{T}$ & $\mathrm{T}$ & $\mathrm{T}$ & $\mathrm{T}$ & $\mathrm{T}$ & * & C \\
\hline PON2/rs4729189 & $\mathrm{T}$ & $\mathrm{T}$ & $\mathrm{T}$ & A & A & A & * & A \\
\hline PON2/rs10261470 & A & A & G & G & G & G & * & G \\
\hline PON2/rs2299267 & A & A & A & A & G & A & * & A \\
\hline PON2/rs17876183 & G & G & G & G & G & G & * & G \\
\hline Haplotype Frequency & 0.073 & 0.051 & 0.052 & 0.052 & 0.096 & 0.192 & 0.161 & 0.323 \\
\hline Coefficient & 0.21 & 1.14 & 0.31 & 0.47 & -0.57 & 1.66 & 0.06 & \\
\hline Standard Error & 0.56 & 0.72 & 0.68 & 0.70 & 0.80 & 0.41 & 0.44 & \\
\hline T statistic & 0.38 & 1.59 & 0.46 & 0.67 & -0.72 & 4.04 & 0.14 & \\
\hline$P$ & 0.705 & 0.113 & 0.643 & 0.506 & 0.475 & $6.1 \times 10^{-5}$ & 0.890 & \\
\hline
\end{tabular}

stepwise regression analysis of PON2 genotypes and indicates that no specific haplotype influences PON activity independent of the analyzed PON2 genotypes.

\section{PON2 tagSNPs and SLE risk}

We examined the relation of 14 PON2 tagSNPs with SLE risk through single-site and haplotype analyses. Four PON2 tagSNPs had less than 5 individuals who were homozygous for the minor allelle: PON2/ rs13306702, PON2/rs17876116, PON2/rs17876183 and PON2/rs17876205. For these SNPs, the rare homozygotes were combined with the heterozygote individuals for the association analysis with SLE risk (dominant model). The remaining SNPs (MAF range: $\sim 10-45 \%$ ) were analyzed under the additive model for which we had $80 \%$ power to detect a minimum OR of $\sim 1.5-1.3$ at the $5 \%$ significance level. In single-site analysis, the genotype frequencies of all PON2 tagSNPs were comparable between cases and controls. Like single-site, no significant association was found with the 14-site PON2 haplotype analysis (data not shown).

\section{PON2 tagSNPs and SLE-related clinical manifestations}

In addition to SLE risk, we examined the association of 14 PON2 tagSNPs with seven SLE-related subphenotypes within the SLE sample, by stratifying the SLE cases for the presence and absence of the subphenotype of interest and using a dominant model. Three PON2 tagSNPs (rs11981433, rs17876183, and rs17876205) showed modest association with lupus nephritis (age and recruitment site adjusted $p$-values ranging between 0.013 0.032). The genotype distributions of these SNPs in SLE cases with/without nephritis and the age and recruitment site adjusted ORs were as follows; for rs11981433 TT: 43.80\%/33.68\% \& TC+CC: $56.20 \% / 66.32 \%$ (OR: 0.61, $95 \%$ CI: 0.39-0.96, $P=0.032$ ), for rs17876183 GG: $93.50 \% /$ 98.25\% \& GA+AA: 6.50\%/1.75\% (OR: 4.63, 95\% CI: $1.37-$ $15.59, P=0.013)$, and for rs17876205 GG: $93.50 \% /$ 98.24\% \& GC+CC: 6.50\%/1.76\% (OR: 4.09, 95\% CI: $1.26-$ $13.24, P=0.019)$. One of these SNPs, rs11981433, showed also modest association with immunologic disorder in the same direction as observed for lupus nephritis (TT: 39.66\%/29.31\% \& TC+CC: $60.34 \% / 70.69 \%$, adjusted OR: 0.62 , 95\% CI: 0.39-0.98, $P=0.042)$. No other significant associations were observed between PON2 tagSNPs and SLE-related subphenotypes evaluated in this study.

\section{Discussion}

Inter-individual variation in serum PON activity is under strong genetic influence and PON1 genetic variants appear to be the main contributors $[7,9]$. We have previously shown that in addition to PON1 SNPs, the PON3 SNPs also influence serum PON activity, although at a lesser degree [7,21]. Altogether PON1/rs662 (p. Gln192Arg), PON1/rs854560 (p.Leu55Met), PON3/ rs740264 and $P O N 3 /$ rs17884563 SNPs account for more than $40 \%$ of variation in serum PON activity among Caucasians $[7,21]$. Since the impact of PON2 SNPs on 
PON activity has not been comprehensively evaluated to date, we examined the role of PON2 tagSNPs in regulation of serum PON activity while taking also into account the effects of the SNPs in PON1 and PON3 genes that lie in close vicinity of PON2.

We found significant associations of one coding and four non-coding PON2 tagSNPs, [rs6954345 (p. Ser311Cys), rs13306702, rs987539, rs11982486, and rs4729189 with $p$-values ranging from 0.005 to $2.1 \times 10^{-6}$ ] with PON activity, after incorporating effects of relevant PON1 and PON3 SNPs as well as environmental covariates. The overall contribution of $P O N 2$ genetic variation on PON activity was $3.4 \%$. A similar association of the PON2/rs6954345 (p.Ser311Cys) SNP with serum PON activity in the same direction (minor allele associated with increased PON activity) has also been reported by Mackness et al. (2000) in type II diabetic individuals [26]. Although PON2/rs6954345 (p.Ser311Cys) SNP is highly correlated with $P O N 3 /$ rs740264 SNP which is a known modifier of PON activity $\left(r^{2}=0.77\right.$; see the second paragraph in LD analysis results), the observation of 4 additional independently and highly significantly associated PON2 tagSNPs supports a possible independent effect of $P O N 2$ genetic variation on PON activity.

The lower contribution of PON2 on PON activity as compared to PON1 and PON3 is likely due to the fact that unlike PON1 and PON3 proteins that are bound to circulating HDL in the blood, PON2 protein is believed to be predominantly intracellular and membrane bound [27-30]. The observed effect on PON activity may be related to a low level secretion of PON2 enzyme into blood [27] and/or the presence of a low-abundant secreted PON2 isoform. $\mathrm{Ng}$ et al. (2001) [27] failed to detect PON2 protein secretion by Western analysis despite the presence of a "putative consensus signal sequence" in PON2 N-terminal region (similar to that in PON1 and PON3), therefore they argued that the protein could either be subject to rapid degradation following its secretion or secreted at a very low level. Although most studies mainly focused on two PON2 isoforms [29,31], Mochizuki et al. (1998) [32] reported that alternative splicing of PON2 transcript results in several PON2 splice forms. Nevertheless, our results indicate only a small effect of PON2 variation on serum PON activity and confirms that the circulatory HDL-bound PON1 is the major modulator. Lack of complete knowledge regarding the natural substrate for PON2 enzyme is a major limiting factor in evaluating the role of PON2 genetic variation in PON2 enzymatic activities.

Although several studies linked PON2 variants to various diseases like amyotrophic lateral sclerosis [33,34], Alzheimer's disease [35], CHD [14-16], and metabolic traits [18] with controversial findings [36], there is no published study that links PON2 with SLE risk. Regardless of the association observed between PON2 variants and PON activity, we did not find any major contribution of PON2 genetic variation to overall SLE risk neither in single-site or haplotype analyses. This is consistent with our prior observations regarding the relation between PON1 and PON3 SNPs and SLE risk [7,21].

Lupus nephritis is a major cause of mortality in SLE patients [1]. Some studies reported associations of PON2 genetic variation with renal dysfunction or nephropathy $[17,37]$. Our group previously reported modest associations of three PON1 promoter SNPs with lupus nephritis in Caucasian SLE patients [7]. Similarly, we observed modest associations of PON2 SNPs with lupus nephritis in Caucasian SLE cases (rs11981433, rs17876183 and 17876205 with covariate-adjusted $p$-values ranging between 0.013-0.032). Our pairwise LD analysis of the nephritis-associated three PON1 promoter SNPs [7] (rs705379, rs705381 and rs854573) and three PON2 SNPs did not reveal high correlations between these SNPs (data not shown). A large prospective study [17] comprising 3,374 diabetic subjects evaluated PON2 variations with indices of renal dysfunction and reported the association of PON2/rs6954345 (p.Ser311Cys) and rs12704795 SNPs with renal dysfunction. Although no association was observed with PON2/ rs6954345 (p.Ser311Cys) SNP and lupus nephritis in our study, significant association was found with PON2/ rs11981433 SNP that is highly correlated with PON2/ rs12704795 SNP $\left(r^{2}=0.98\right)$. Our results also revealed a modest association of $P O N 2 / \mathrm{rs} 11981433$ SNP with immunologic disorder (adjusted $P=0.042$ ). Although the associations observed with SLE subphenotypes were not strong enough to survive correction for multiple testing, the association of rs11981433 SNP with more than one SLE subphenotype in the same direction (protective) would argue in favor of its possible genuine effect on SLE-related clinical features.

\section{Conclusions}

In summary, our results suggest that PON2 genetic variation is significantly and independently associated with variation in serum PON activity (after incorporating effects of age, SLE status, relevant PON1 and PON3 variation), although to a much lesser extent than PON1. We found no obvious association between PON2 tagSNPs and SLE risk, however, we observed few modest associations with lupus nephritis and SLE-related immunological disorder which await replication in larger independent samples. A comprehensive resequencing and association analysis of $P O N$ gene cluster may help to better understand extent to which this locus contributes to the regulation of PON activity and to the risk of SLE-related clinical features. 


\section{Additional material}

Additional file 1: Table S1. PCR and Sequencing primers used for PON2 SNPs genotyped by Pyrosequencing

\section{Acknowledgements}

This study was supported by the National Institutes of Health grants R01HL074165, R01-HL088648, R01-AR046588, K24-AR002213, K24-AR002138, and P60-AR48098.

\section{Author details}

${ }^{1}$ Department of Human Genetics, University of Pittsburgh, Pittsburgh, PA, USA. ${ }^{2}$ Division of Rheumatology and Clinical Immunology, Lupus Center of Excellence, Pittsburgh, PA, USA. ${ }^{3}$ Division of Rheumatology, Feinberg School of Medicine, Northwestern University, Chicago, IL, USA. ${ }^{4}$ West Penn Allegheny, Pittsburgh, PA, USA

\section{Authors' contributions}

Author's Contributions were as follows: study conception and design (MIK), acquisition of clinical data (AHK, EYR, RRG, SM), acquisition of PON2 genetic data (SD), assistance for acquisition of genetic data (FYD, MIK), molecular and/or statistical analysis and interpretation of the data (SD, FYD, ASD, CMK, MIK), drafting of the manuscript (SD, FYD, CMK, MIK), critical revision and final approval of the manuscript (all authors).

\section{Competing interests}

The authors declare that they have no competing interests.

Received: 23 July 2010 Accepted: 11 January 2011

Published: 11 January 2011

\section{References}

1. D'Cruz DP, Khamashta MA, Hughes GR: Systemic lupus erythematosus. Lancet 2007, 369:587-596.

2. Lawrence JS, Martins CL, Drake GL: A family survey of lupus erythematosus. 1. Heritability. J Rheumatol 1987, 14:913-921.

3. Manzi S, Meilahn EN, Rairie JE, Conte CG, Medsger TA Jr, JansenMcWilliams L, D'Agostino RB, Kuller LH: Age-specific incidence rates of myocardial infarction and angina in women with systemic lupus erythematosus: comparison with the Framingham Study. Am J Epidemiol 1997, 145:408-415.

4. Ng CJ, Shih DM, Hama SY, Villa N, Navab M, Reddy ST: The paraoxonase gene family and atherosclerosis. Free Radic Biol Med 2005, 38:153-163.

5. Mackness B, Davies GK, Turkie W, Lee E, Roberts DH, Hill E, Roberts C, Durrington PN, Mackness Ml: Paraoxonase status in coronary heart disease: are activity and concentration more important than genotype? Arterioscler Thromb Vasc Biol 2001, 21:1451-1457.

6. Ayub A, Mackness MI, Arrol S, Mackness B, Patel J, Durrington PN: Serum paraoxonase after myocardial infarction. Arterioscler Thromb Vasc Biol 1999, 19:330-335.

7. Tripi LM, Manzi S, Chen Q, Kenney M, Shaw P, Kao A, Bontempo F, Kammerer C, Kamboh Ml: Relationship of serum paraoxonase 1 activity and paraoxonase 1 genotype to risk of systemic lupus erythematosus. Arthritis Rheum 2006, 54:1928-1939.

8. Kiss E, Seres I, Tarr T, Kocsis Z, Szegedi G, Paragh G: Reduced paraoxonase1 activity is a risk for atherosclerosis in patients with systemic lupus erythematosus. Ann N Y Acad Sci 2007, 1108:83-91.

9. Carlson CS, Heagerty PJ, Hatsukami TS, Richter RJ, Ranchalis J, Lewis J, Bacus TJ, McKinstry LA, Schellenberg GD, Rieder M, Nickerson D, Furlong CE, Chait A, Jarvik GP: TagSNP analyses of the PON gene cluster: effects on PON1 activity, LDL oxidative susceptibility, and vascular disease. J Lipid Res 2006, 47:1014-1024.

10. Primo-Parmo SL, Sorenson RC, Teiber J, La Du BN: The human serum paraoxonase/arylesterase gene (PON1) is one member of a multigene family. Genomics 1996, 33:498-507.

11. Gaffney PM, Ortmann WA, Selby SA, Shark KB, Ockenden TC, Rohlf KE, Walgrave NL, Boyum WP, Malmgren ML, Miller ME, Kearns GM, Messner RP,
King RA, Rich SS, Behrens TW: Genome screening in human systemic lupus erythematosus: results from a second Minnesota cohort and combined analyses of 187 sib-pair families. Am J Hum Genet 2000, 66:547-556.

12. Draganov DI, Teiber JF, Speelman A, Osawa $Y$, Sunahara R, La Du BN: Human paraoxonases (PON1, PON2, and PON3) are lactonases with overlapping and distinct substrate specificities. J Lipid Res 2005, 46:1239-1247.

13. Augustinsson KB: The evolution of esterases in vertebrates. Homologous Enzymes and Biochemical Evolution.Edited by: van Thoai N, Roche J. Gordon 1968:299-311.

14. Sanghera DK, Aston CE, Saha N, Kamboh MI: DNA polymorphisms in two paraoxonase genes (PON1 and PON2) are associated with the risk of coronary heart disease. Am J Hum Genet 1998, 62:36-44.

15. Chen $Q$, Reis SE, Kammerer CM, McNamara DM, Holubkov R, Sharaf BL, Sopko G, Pauly DF, Merz CN, Kamboh MI, WISE Study Group: Association between the severity of angiographic coronary artery disease and paraoxonase gene polymorphisms in the National Heart, Lung, and Blood Institute-sponsored Women's Ischemia Syndrome Evaluation (WISE) study. Am J Hum Genet 2003, 72:13-22.

16. Leus FR, Zwart M, Kastelein JJ, Voorbij HA: PON2 gene variants are associated with clinical manifestations of cardiovascular disease in familial hypercholesterolemia patients. Atherosclerosis 2001, 154:641-649.

17. Calle R, McCarthy MI, Banerjee P, Zeggini E, Cull CA, Thorne KI, Wiltshire S, Terra S, Meyer D, Richmond J, Mancuso J, Milos P, Fryburg D, Holman RR: Paraoxonase 2 (PON2) polymorphisms and development of renal dysfunction in type 2 diabetes: UKPDS 76. Diabetologia 2006, 49:2892-2899.

18. Boright AP, Connelly PW, Brunt JH, Scherer SW, Tsui LC, Hegele RA: Genetic variation in paraoxonase- 1 and paraoxonase- 2 is associated with variation in plasma lipoproteins in Alberta Hutterites. Atherosclerosis 1998, 139:131-136.

19. Stoltz DA, Ozer EA, Ng CJ, Yu JM, Reddy ST, Lusis AJ, Bourquard N, Parsek MR, Zabner J, Shih DM: Paraoxonase-2 deficiency enhances Pseudomonas aeruginosa quorum sensing in murine tracheal epithelia. Am J Physiol Lung Cell Mol Physiol 2007, 292:L852-860.

20. Stoltz DA, Ozer EA, Recker TJ, Estin M, Yang X, Shih DM, Lusis AJ, Zabner J: A common mutation in paraoxonase- 2 results in impaired lactonase activity. J Biol Chem 2009, 284:35564-35571.

21. Sanghera DK, Manzi S, Minster RL, Shaw P, Kao A, Bontempo F, Kamboh Ml: Genetic variation in the paraoxonase-3 (PON3) gene is associated with serum PON1 activity. Ann Hum Genet 2008, 72:72-81.

22. Selzer F, Sutton-Tyrrell K, Fitzgerald S, Tracy R, Kuller L, Manzi S: Vascular stiffness in women with systemic lupus erythematosus. Hypertension 2001, 37:1075-1082.

23. Rhew EY, Manzi SM, Dyer AR, Kao AH, Danchenko N, Barinas-Mitchell E Sutton-Tyrrell K, McPherson DD, Pearce W, Edmundowicz D, Kondos GT, Ramsey-Goldman R: Differences in subclinical cardiovascular disease between African American and Caucasian women with systemic lupus erythematosus. Trans/ Res 2009, 153:51-59.

24. Tan EM, Cohen AS, Fries JF, Masi AT, McShane DJ, Rothfield NF, Schaller JG, Talal N, Winchester RJ: The 1982 revised criteria for the classification of systemic lupus erythematosus. Arthritis Rheum 1982, 25:1271-1277.

25. Hochberg MC: Updating the American College of Rheumatology revised criteria for the classification of systemic lupus erythematosus. Arthritis Rheum 1997, 40:1725.

26. Mackness B, Durrington PN, Abuashia B, Boulton AJ, Mackness MI: Low paraoxonase activity in type II diabetes mellitus complicated by retinopathy. Clin Sci 2000, 98:355-363.

27. Ng CJ, Wadleigh DJ, Gangopadhyay A, Hama S, Grijalva VR, Navab M, Fogelman AM, Reddy ST: Paraoxonase-2 is a ubiquitously expressed protein with antioxidant properties and is capable of preventing cellmediated oxidative modification of low density lipoprotein. J Biol Chem 2001, 276:44444-44449.

28. Ng CJ, Hama SY, Bourquard N, Navab M, Reddy ST: Adenovirus mediated expression of human paraoxonase 2 protects against the development of atherosclerosis in apolipoprotein E-deficient mice. Mol Genet Metab 2006, 89:368-373.

29. Horke S, Witte I, Wilgenbus P, Krüger M, Strand D, Förstermann U: Paraoxonase-2 reduces oxidative stress in vascular cells and decreases endoplasmic reticulum stress-induced caspase activation. Circulation 2007, 115:2055-2064 
30. Levy E, Trudel K, Bendayan M, Seidman E, Delvin E, Elchebly M, Lavoie JC, Precourt LP, Amre D, Sinnett D: Biological role, protein expression, subcellular localization, and oxidative stress response of paraoxonase 2 in the intestine of humans and rats. Am J Physiol Gastrointest Liver Physiol 2007, 293:G1252-1261.

31. Shamir R, Hartman C, Karry R, Pavlotzky E, Eliakim R, Lachter J, Suissa A, Aviram M: Paraoxonases (PONs) 1, 2, and 3 are expressed in human and mouse gastrointestinal tract and in Caco- 2 cell line: selective secretion of PON1 and PON2. Free Radic Biol Med 2005, 39:336-344.

32. Mochizuki H, Scherer SW, Xi T, Nickle DC, Majer M, Huizenga JJ, Tsui LC, Prochazka M: Human PON2 gene at 7q21.3: cloning, multiple mRNA forms, and missense polymorphisms in the coding sequence. Gene 1998, 213:149-157

33. Valdmanis PN, Kabashi E, Dyck A, Hince $P$, Lee J, Dion P, D'Amour M, Souchon F, Bouchard JP, Salachas F, Meininger V, Andersen PM, Camu W, Dupré N, Rouleau GA: Association of paraoxonase gene cluster polymorphisms with ALS in France, Quebec, and Sweden. Neurology 2008, 71:514-520.

34. Saeed M, Siddique N, Hung WY, Usacheva E, Liu E, Sufit RL, Heller SL, Haines JL, Pericak-Vance $M$, Siddique T: Paraoxonase cluster polymorphisms are associated with sporadic ALS. Neurology 2006 67:771-776.

35. Janka Z, Juhász A, Rimanóczy AA, Boda K, Márki-Zay J, Kálmán J: Codon 311 (Cys $\mathbb{}$ Ser) polymorphism of paraoxonase-2 gene is associated with apolipoprotein E4 allele in both Alzheimer's and vascular dementias. Mol Psychiatry 2002, 7:110-112.

36. Wheeler JG, Keavney BD, Watkins $H$, Collins $R$, Danesh J: Four paraoxonase gene polymorphisms in 11212 cases of coronary heart disease and 12786 controls: meta-analysis of 43 studies. Lancet 2004, 363:689-695.

37. Pinizzotto M, Castillo E, Fiaux M, Temler E, Gaillard RC, Ruiz J: Paraoxonase2 polymorphisms are associated with nephropathy in Type II diabetes. Diabetologia 2001, 44:104-107.

\section{Pre-publication history}

The pre-publication history for this paper can be accessed here: http://www.biomedcentral.com/1471-2350/12/7/prepub

doi:10.1186/1471-2350-12-7

Cite this article as: Dasgupta et al:: Association analysis of PON2 genetic variants with serum paraoxonase activity and systemic lupus erythematosus. BMC Medical Genetics 2011 12:7.

\section{Submit your next manuscript to BioMed Central and take full advantage of:}

- Convenient online submission

- Thorough peer review

- No space constraints or color figure charges

- Immediate publication on acceptance

- Inclusion in PubMed, CAS, Scopus and Google Scholar

- Research which is freely available for redistribution

Submit your manuscript at www.biomedcentral.com/submit 